\title{
Cocina, creatividad y cultura en el pacífico potamoral en perspectiva de etnoeducación en derechos humanos*
}

\section{Kitchen, creativity and culture in the potamoral pacific under an ethnoeducation in human rights perspective}

PP. 88-100

Carlos Alberto Velasco díaz **

REC: $20 / 05 / 2020$

ACEP: $15 / 09 / 2020$

\section{Resumen}

Los estudios sobre los africanos y sus descendientes parten tradicionalmente de la esclavización de estos pueblos, con lo que a lavez invisibilizan la esclavitud de los pueblos de piel clara. Dicha estrechez cambia automáticamente el molde para estudiar estas culturas. El objeto de este artículo es relievar el papel que ha jugado la cocina y sobre todo las mujeres en muchos procesos integrantes de ese gran proyecto libertario que nace desde el momento de su secuestro en las costas occidentales del continente madre. Mediante la utilización de diferentes métodos para aproximarse al tema de la cocina como producto de la cultura y como expresión de creatividad de los pueblos afros -que van desde la observación directa, observación participante, historias de vida, entrevistas, conversatorios dentro de una metodología cualitativa- permitieron cosmocer, -no conocer- este componente tan importante en la vida de los pueblos afros y recuperar platos inventados por las esclavizadas llamados el

* Artículo producto de investigación proyecto de investigación "Etnoeducación, derechos humanos e interculturalidad" Fundación Universitaria Católica Lumen Gentium. 2019.

** Doctor en Humanidades, línea de Historias, Sociedades y Culturas Afrolatinoamericanas de la Universidad del Valle.

Correo electrónico: cavelascod@unicatolica.edu.co. Orcid: https://orcid.org/oooo-0002-9115-6590. 
“Caigamos juntos", la chicha de pipilongo y el pan de abonar o pan de abono -por deformidad dialéctica se le llama hoy pandebono-.

Los productos en mención son parte de la creatividad, de las invenciones realizadas en medio de la negación en el contexto socioeconómico de las haciendas esclavistas ubicadas en la jurisdicción del antiguo cantón de Caloto, deben ser estudiadas desde su creatividad y no simplemente vistas en esa relación tradicional de cosificación como esclavizados que no permitieron ver en ellos seres creativos e inventivos. Estas tareas se deben asumir dentro de las actividades de la etnoeducación entendida en sus cuatro componentes: el etnohistórico -la inclusión de los grupos subalternizados y racializados-; etnopedagógico, -cátedra de estudios afrocolombianos-; etnosocial o de desarrollo comunitario; y el componente etnocultural.

Palabras claves: creatividad afro, cocina, cultura, cultura afrocolombiana, pacífico potamoral.

\section{Abstract}

Studies on Africans and their descendants have traditionally started from the enslavement of these peoples, while making the slavery of light-skinned peoples invisible. This narrowness automatically changes the mold for studying these cultures. The purpose of this article is to highlight the role that cuisine and especially women have played in many processes that are part of this great libertarian project that was born from the moment of their kidnapping on the western coasts of the mother continent. Through the use of different methods to approach the subject of cuisine as a product of culture and as an expression of creativity of Afro peoples -ranging from direct observation, participant observation, life stories, interviews, conversations within a qualitative methodology- allowed to cosmocer, -not to acknowledge- this component so important in the life of the Afro peoples, and to recover dishes invented by the enslaved women called the "let's fall together", the chicha de pipilongo and the bread of fertilizer, -due to dialectical deformity, today it is called pandebono-.

The products in question are part of the creativity, of the inventions made in the midst of the denial in the socioeconomic context of the slave farms located in the jurisdiction of the former canton of Caloto. They must be studied starting from their creativity and not simply seen in that traditional relationship of objectification as slaves, which did not allow to see them as creative and inventive beings. These tasks must be assumed within the activities of ethnoeducation understood in its four components: the ethnohistorical the inclusion of subalternized and racialized groups-; ethnopedagogical -Lectures on AfroColombian studies-; ethnosocial or community development; and the ethnocultural component.

Keywords: afro creativity, cooking, culture, Afro-Colombian culture, peaceful potamoral.

\section{Introducción}

La pretensión de este artículo de investigación es navegar un poco con elementos muy sencillos en el basto mundo de las sabidurías de los pueblos afrocolombianos. Con ello ha sido posible visibilizar los saberes de los africanos en el momento de llegada al suelo americano, en contraposición al pensamiento occidental decimonónico que quiso, con pseudoteorías, mostrar lo contrario.

El antropólogo Patricio Guerrero Arias (2018) pone en el centro del debate la tensión entre conocimiento y sabiduría; la primera la ve como una noción desarrollada e impuesta 
por la racionalidad occidental que estudia al mundo solo desde el campo "cognitivo" de la racionalización de occidente, mientras que la mirada desde las sabidurías ancestrales conmina a reformular nociones desarrolladas por la racionalidad. Desde esta perspectiva se habla de un universo en vez de un bioverso en el cual la vida es el centro -biocentrismo-; en el reconocimiento de todos los seres habitantes de la naturaleza, contrariamente se habla de cosmovisión, privilegia así solo el sentido oculocéntrico y descarta los otros como el tacto, el olfato, oído, gusto, y centra todo en la vista, por eso se prefiere aquí referirse a cosmocimientos.

Desde esta perspectiva, las comunidades afrocolombianas en el pacífico potamoral ${ }^{1}$ han desarrollado muchas sabidurías reflejadas en su cosmoexistencia como pueblo afro dentro del marco de la filosofía del Muntu.

\section{Materiales y metodología}

El proyecto de investigación Etnoeducación e Interculturalidad que desarrolla la Facultad de Educación, a través de los programas de Licenciatura en Ciencias Sociales y Especialización en Educación en Derechos Humanos en Unicatólica hace un acercamiento a la conceptualización de los derechos humanos con el propósito de lograr su ampliación desde la perspectiva de la matriz de necesidades y satisfactores propuesta por Manfred Max-Neef (2019),
Antonio Elizalde y Martin Hopenhayn de la Cepaur. Les ubica en las categorías existenciales y axiológicas, pero fundamentalmente en las nueve categorías axiológicas. La cocina vista como producto cultural -tema que nos convoca- hace parte integrante de los satisfactores. Desde esta perspectiva, tanto las necesidades como los satisfactores se estudian como derechos humanos; un elemento como la cocina es parte integrante de las necesidades axiológica de identidad. No obstante, al estudiarla como satisfactor se encuentra que funciona como satisfactor sinérgico de varias necesidades, tales como participación, subsistencia e identidad.

En el proceso de investigación para la reconstrucción de algunos platos componentes de la cocina tradicional de la región del pacífico potamoral se realizaron conversatorios con diferentes comunidades, principalmente con mujeres, las cuales son depositarias de gran parte de la tradición. La observación participante es un método efectivo cuando se trabaja la cocina como producto cultural en las comunidades afrocolombianas. De la misma manera, la revisión bibliográfica de publicaciones hechas sobre este componente en la zona de estudio, suministraron elementos importantes para esta investigación, no obstante, la relación establecida en otros contextos, pero que guardan estrecha relación con la cocina como producto cultural. 
Figura 1.

El pacífico potamoral, de la invisibilidad al reconocimiento de las sabidurías

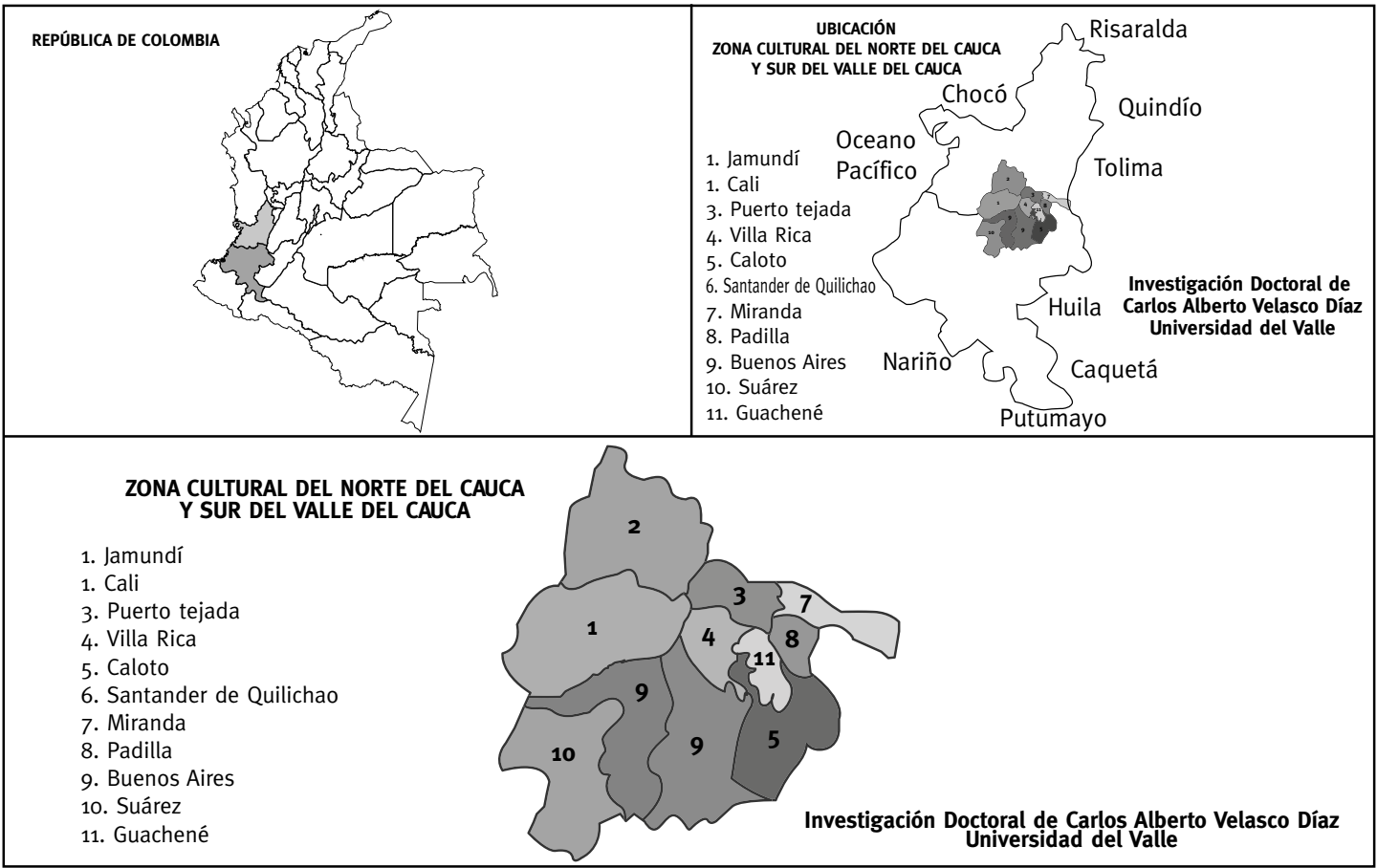

Fuente: Desarrollo del autor

Potamoral es una noción que se origina en la reflexión de la tesis doctoral y se adoptó para denominar a la zona cultural del norte del departamento del Cauca y sur del departamento delValle del Cauca-cuna de los violinescaucanos-, integrada por diez espacialidades, la cual es parte de la región pacífica colombiana integrada por dos subregiones: el litoral y el potamoral y se entiende la potamología como la ciencia que estudia esa relación de la selva con los ríos.

\section{Mujeres y hombres, de la negación a la construcción de afrosabidurías}

Las sabidurías se refieren a un saber vivido, no son un simple conocimiento, sino cosmocimiento del bioverso y no universo ${ }^{2}$. Son las sabidurías de los pueblos afros y no la racionalidad la que permite explicar hoy por qué subsisten creatividades milenarias como la medicina tradicional; además, muchas de sus guardianas no saben leer ni escribir el alfabeto español; también nos muestra que estas comunidades no han estado sordas a los ecos de las sabidurías en contraposición a la lógica de la racionalidad.

\section{¿Conocimientos o cosmocimientos, racionalidad o sabidurías?}

En el proceso de colonialidad del ser, poder y saber se identifica que construir conceptos displicentes para las culturas africanas dentro de las pocas invenciones, a partir de las limitaciones intelectuales de los europeos que invadieron a América, aparece la palabra 'negro' que etimológicamente significa cadáver. Con ello

2 La noción de universo, propia de la racionalización occidental, es una sola fuerza, la vida es el resultado de una sola fuerza y tiene una sola dimensión, uni-verso. La noción de bioverso desde la propuesta de Arias decoloniza el sentido universalista que impone la cosmología occidental en la que el centro es el hombre, acá es la vida; somos parte del bioverso en el cual todo está vivo y se busca la articulación desde el corazonar con ese centro. Según este investigador, esta noción ha recibido varios nombre:, pariverso, multiverso, pluriverso; la diferencia es que el bio-verso pone en el centro la vida y no a la persona, pone como principio de todo lo existente en el cosmos, la vida, de allí que la sabiduría no es un simple conocimiento sino cosmocimiento del bioverso (Guerrero Arias, 2018). 
se reflejan no solo connotaciones racistas, sino también el mar de ignorancia que circundaba a los europeos. Hayunas diferenciasmuymarcadasentre la epistemología desde el pensamiento occidental y las sabidurías de los pueblos subalternizados, como se observa en la siguiente matriz:

\section{Tabla 1.}

Cuadro comparativo entre las sabidurías $y$ epistemologías

\begin{tabular}{ll}
\hline \multicolumn{1}{c}{ Epistemología } & \multicolumn{1}{c}{ Sabiduría } \\
\hline El fundamentalismo carte- Siento, pienso y hago. \\
siano dice: “Pienso, luego \\
existo".
\end{tabular}

Construye una cosmovisión, Cosmoexistencia, cosuna manera de ver cogniti- movivencia, una forma vamente el mundo y la vida, de ser viviendo parte lo que evidencia un sentido del tejido cósmico de la oculocentrista. vida.

Conocimiento para el tener, Cosmocimiento para que no ha hecho ni mejores el ser, permitiendo la seres humanos, ni más realización humana y a felices. nuestra felicidad.

Prioriza la acumulación de Prioriza la vida y no la capital sobre la vida. acumulación de riqueza.

El episteme busca explicar, La sabiduría busca analizar, hipotetizar teori- comprender viviendo, zando. por eso nos transforma.

Arrogancia, vanidad. Sencillez, humildad.

Conocimiento carente de Cosmocimiento que se espiritualidad, desacraliza sostiene en una profunla vida, la naturaleza para da espiritualidad que transformarla en objeto de busca el lazo sagrado dominio. que nos une con la vida y nuestro lugar en el cosmos.

Se sustenta en la hegemonía No hay métodos hegede la inducción y la mónicos, contempla la deducción como métodos de abducción, como otro conocimiento. miento. Da espacio a la intuición, la imaginación, la creatividad, se aprende desde la alegría.

Fuente: adaptado de la matriz de Guerrero Arias (2018)

La sabiduría como cosmocimiento, tiene en cuenta y les otorga validez a los sentidos de tacto, olfato, oído, gusto y vista. Para las culturas afros también es en el corazón donde surge la espiritualidad y la sabiduría, supone hablar de un cosmos creado al ritmo de tambores para que recordar así el latido del corazón de la vida (Guerrero Arias, 2018. p. 271).

\section{Sabidurías, cocina y cultura}

\section{Figura 2.}

Fotografía de Ana Patricia Chávez Preciado, chef afro internacional

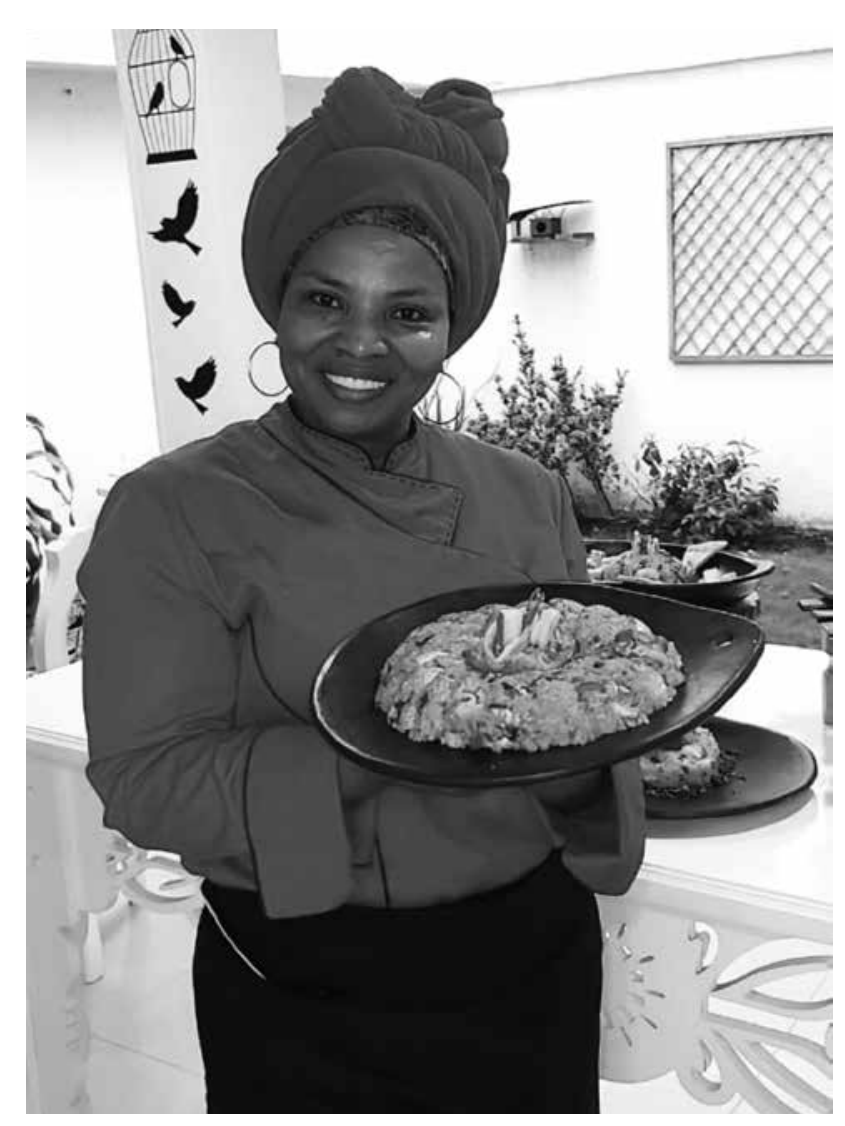

Fuente: archivo de Carlos Alberto Velasco Díaz.

El presente componente tiene que ver con la producción, el arte del cultivo, formas de combinación de los productos de la zona y de la diáspora en la preparación de sustancias alimenticias. Las prácticas culinarias, variedad de recetas, articulaciones con las sonoridades, 
procesos emancipatorios y con las sabidurías kemíticas ${ }^{3}$, nombres de platos, productos que se utilizan, maneras de sazonar y conservar los alimentos se incluyen allí. También los significados simbólicos y medicinales de los alimentos. No es hablar de gastronomía porque no se comparte el uso del término con otros investigadores por considerarlo de uso errático; la comida no se disfruta en el estómago o región gástrica y en ese sentido se prefiere aproximar el tema a manera de cocina como producto de la cultura.

\section{Diáspora botánica y sabidurías}

Debemos tener en cuenta el estudio de Judith Carney y Rosa Acevedo Marín (2003) sobre las plantas de la diáspora africana en la botánica americana de la fase colonial. Esta muestra cómo las poblaciones esclavizadas contribuyeron al establecimiento en Las Américas de productos alimenticios y plantas medicinales de origen africano. Todo no llegó de Europa como lo han querido plantear en su concepción eurocéntrica monolítica; de África llegó una multiplicidad de sabidurías, como lo plantean las investigadoras:

En su mayoría, estas plantas cruzaron el Atlántico en los cargamentos de barcos negreros como provisiones alimenticias, productos medicinales o para usos generales. Después de resistir largos viajes transatlánticos, estas plantas fueron cultivadas en las áreas de siembra de subsistencia de los esclavos, en huertas caseras y en los sitios de habitación por los negros libertos (Carney y Acevedo Marín, 2003).

Las mujeres en la ruta transatlántica utilizaron muchas estrategias para la reconstrucción de la vida en el extraño continente. Una de estas leyendas narra, por ejemplo, la astucia y el heroísmo de una esclavizada que al dejar África llevó consigo granos de arroz escondidos en sus cabellos (Carney y Acevedo, 2003). Este hecho nos demuestra que no era nueva la práctica de las mujeres esclavizadas en las haciendas del Pacífico potamoral de almacenar semillas y oro en sus peinados o trisnejas.

\section{Figura 3.}

Foto de uno de los peinados de la zona

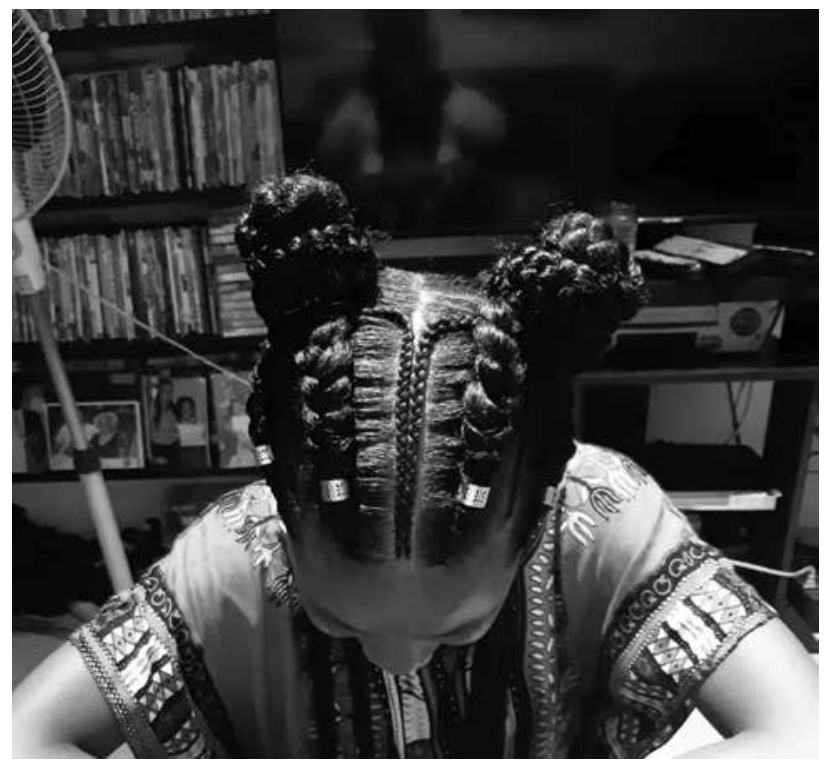

Fuente: elaboración propia (2019)

Todo este vasto cosmocimiento en las ciencias botánicasqueteníanlaspoblacionesesclavizadas se utilizaron para distintas actividades en el nuevo territorio: en la alimentación, en defensa de su libertad (recuérdese que envenenaron y asesinaron a muchos esclavistas). Desde esta perspectiva, en el proceso de invisibilización de los pueblos africanos y en la estructuración del paradigma eurocéntrico se trató de negar todo ese aporte de los afros a nivel de las ciencias.

En el siguiente cuadro se nombran algunas de las plantas investigadas por Carney y Acevedo y encontradas en el pacífico potamoral.

3 Kemíticas es un término que adoptado en la tesis doctoral del autor para referenciar las sabidurías bioquímicas en las cuales se movieron los pueblos africanos traídos como secuestrados a las tierras americanas. Kemi viene del wolof y significa negro u oscuro, es la raíz de la palabra química, según las investigaciones de Cheikh Anta Diop. 
Tabla 2.

Plantas de la diáspora

\begin{tabular}{ll}
\hline \multicolumn{2}{l}{$\begin{array}{l}\text { Plantas de la Diáspora Africana de uso en la alimen- } \\
\text { tación de la zona cultural norte del Cauca y Sur del } \\
\text { Valle del Cauca }\end{array}$} \\
\hline Nombre vulgar & Nombre científico \\
\hline Sorgo & Sorghum bicolor \\
\hline Sandía & Citrullus lanatus \\
\hline Arroz & Oriza glaberrima \\
\hline Tomate africano & Solanum aethiopicum \\
\hline Gundul o frijolito de año & Cajanus cajan \\
\hline Fríjol caupí & Vigna unguiculata \\
\hline Avena etíope & Avena abyssinica \\
\hline Café & Coffea arabaica \\
\hline Sorgo & Sorghum bicolor \\
\hline Sandía & Citrullus lanatus \\
\hline Arroz & Obliza glaberrima \\
\hline Tomate africano & Solanum aethiopicum \\
\hline Gundul o frijolito de año & Cajanus cajan \\
\hline Frijol Caupí & Vigna unguiculata \\
\hline Avena etíope & Avena abyssinica \\
\hline
\end{tabular}

Fuente: Autor adaptado a partir de Carney y Acevedo (2003)

Otra especie de mucho uso en estas comunidades es la conocida como quassia-de uso medicinal-, es un tallo de sabor amargo y lo utilizan para prevenir problemas del hígado. Su nombre origina en Quassi, un esclavo transportado del oeste africano a Surinam, él difundió sus propiedades curativas alrededor de 1730. Cuando esta especie se le mostró a Linnaeus en 1761, la llamó Quass como un tributo a Quassi y de esta forma inmortalizó su contribución (Carney y Acevedo, 2003).

\section{La cocina como producto de la cultura}

Gran diversidad de estas plantas se utilizó en la preparación de alimentos, de allí la importancia de estudiar la cocina como un elemento de la cultura. Mirar la cocina más allá del recetario permite encontrar relaciones sociales, códigos de comunicación y simbolismos (Patiño Ossa, 2007). En cuanto a relaciones sociales, tradicionalmente entre los habitantes se comparten mutuamente comidas en la época de navidad, los jóvenes realizan comilonas o "secas de arroz". Esto se convierte en un pretexto para compartir con otros, se dan enamoramientos, el chisme también juega un papel de control social.

También se identifican códigos de comunicación, simbolismos. Por ejemplo, una sustancia que produce la leña cuando se le somete al fogón se empleaba para untarle en el ombligo a los menores y evitar las hernias umbilicales. Dentro de estos simbolismos se encontró que hay unas cocinas en las que todavía las señoras mantienen una cruz, porque según ellas, los muertos en pena venían a molestar a quien estuviera cocinando (Velasco Díaz, 2011).

\section{Sabidurías desde la extrañeidad}

La llegada a un mundo extraño de las culturas africanas implicó que desde la extrañeidad y mediante la creatividad tuvieron que construir las condiciones mínimas para el mundo que necesitaban. En la elaboración de los utensilios de la cocina nortecaucana - survalluna fueron creativos, inicialmente los construyeron con elementos de la región. Usaron principalmente la guadua, árboles de la finca para elaborar bateas, cagüingas y cucharas; el zumbo o mate para las cucharas e instrumentos musicales; vasijas para orinar, tomar agua y macerar las plantas medicinales.

Los cuchareros elaborados en guadua, con especial precaución de coger la guadua en luna menguante y en horas de la mañana para asegurar mejor la conservación; las cucharas de mate las elaboraron con media totuma, a veces hasta con un cuarto de totuma, se utiliza para servir la comida. las más pequeñas para comer; la tinaja o recipiente de barro cocido se utilizaba para almacenar agua. En muchas casas habían tres tinajas, una la utilizaban 
para almacenar solo el agua de beber, la otra para almacenar agua para los quehaceres de la cocina y la tercera para almacenar las carnes que compraban para toda la semana, la cual partían en tiras y la salaban ante la ausencia de artefactos de refrigeración por la inexistencia del servicio de energía eléctrica; la susunga -es el colador antiguo en la región- la elaboraban con un zumbo partido a la mitad al que le hacían agujeros, era útil porque en ella colaban el maíz para los diferentes preparados y las plantas medicinales; la piedra de moler: una piedra que con el uso se le hacía un hueco en el centro y la utilizaban para moler los aliños, el azafrán de vena y machacaban los plátanos; el molinillo: palo de forma cilíndrica que en la parte inferior era más abultado y dentado. Se utilizaba generalmente para batir el chocolate y donde no había licuadora, para hacer el jugo; la callana que es una paila de barro cocido, de paredes delgadas y se utilizaba para hacer las arepas y para tostar el café para el consumo; la cagüinga: una cuchara grande o cucharón que elaboraban en madera o en guadua y se utilizaba para batir el dulce manjar blanco. La cagüinga pequeña la utilizaban para la preparación de coladas de millo o sorgo, plátano y de la mazamorra.

En la complejidad de la cocina de la zona cultural norte del Cauca y sur del Valle los antepasados africanos utilizaron muchas plantas para darle buen sabor a las comidas, algunas traídas desde el continente africano, otras traídas por los europeos y las que conocen mediante el contacto creativo con los pueblos nativos que habitaban el valle geográfico del río Cauca. En este campo se encuentran algunas medicinales, además muchas se siembran en los patios de las casas como el pipilongo, este aún se cultiva en estos espacios. Entre las plantas más comunes están la rascadera, de la familia de las aráceas. Se le conoce también como mafafa en algunos sectores afros y en los pueblos nativos. Es un tubérculo que se cultiva generalmente en las fincas y en los solares de las casas. Se utiliza en la preparación de sopas y sancocho. Se usa en troncos pequeños o rallada y es el truco de las abuelas para espesar las comidas y darles ese sabor característico de las comidas de la región.

\section{Maíz y creatividad: el pandebono, inven- ción de las africanas en las haciendas}

El pacífico potamoral -norte del Cauca y sur del Valle- es una de las regiones donde se prepara un mayor número de platos con subproductos del maíz. Esto además tiene su razón histórica porque el maíz, según el investigador Patiño (2007) era uno de los productos base de la comida en la región en la época de la colonia.

El pandebono lo crean las mujeres afros en las haciendas del norte del Cauca, según el testimonio de la matrona Josefina Abonía de Mancilla, nacida en 1912 y recogido por la maestra Ana Melba Banguero (s. f.) de la espacialidad de Guachené. En las haciendas de zona las mujeres inventaron un pan de maíz al cual le adicionaron queso y almidón, y al que bautizaron con el nombre de pan de abonar ${ }^{4}$, también le llamaban pan de abono; por comodidad dialectal pegaron las dos palabras y quedó en el imaginario la palabra pandebono.

\section{Sabidurías kemíticas}

Esta sabiduría hace referencia a las capacidades y cosmocimientos en el campo de la química que poseen las culturas al momento de crear o inventar bebidas medicinales y rituales. 


\section{El aguardiente}

Desde el periodo colonial, para las comunidades nortecaucanas el aguardiente guardó un significado tanto social como ritual. Es probable que los primeros africanos que llegaron a laborar en las haciendas del norte del Cauca tuvieran vastos conocimientos de procesos bioquímicos que pusieron al servicio de la economía colonial. Existe en la tradición cultural de la zona un canto denominado trapiche de los demonios, este reconstruye todo el proceso de la caña de azúcar desde la siembra hasta el final de la zafra.

\section{Usos rituales del aguardiente en el pacífico potamoral}

En la zona cultural de estudio el aguardiente ha tenido muchos usos rituales que el europeo, en su poca comprensión de las culturas africanas, afroraizales y afrocolombianas no entendió. Este observaba solo el embriagamiento o las borracheras que, de alguna manera estimularon los levantamientos de esclavizados. No obstante, se usa en el baile ritual del torbellino. Para ello, ubican una botella en el centro del baile y nadie la puede tocar ni tumbar, quien lo haga debe comprar una nueva e igualmente cuando destapan una nueva caneca, el primer trago lo lanzan al suelo, según ellos porque es para las ánimas; consideran que las ánimas -así hayan pasado a otra vida- todavía consumen aguardiente.

En muchas casas mantienen una botella a la cual le adicionan plantas medicinales para curar ciertos males del cuerpo, en otras, especialmente el médico tradicional, como lo platea Ramos, introduce los animales en botellas a las que adiciona plantas medicinales y aguardiente (Ramos, 2014).

En el ritual del bunde y de adoraciones el aguardiente es un elemento importante, facilita las declamaciones, ayuda a vencer la timidez, es un estimulante de la memoria. Cuando las cantoras lo consumen, las letras de los cantos y recitaciones se encadenan mejor en la memoria. Si le adicionan algunas plantas como pino, ajo o cardamomo lo utilizan como desparasitante, no produce guayabo, ni de cabeza ni maluqueras; le adicionan 21 plantas.

El aguardiente en la zona cultural del norte del Cauca y sur del Valle del Cauca ha sido tradicionalmente empleado para las enfermedades del mundo visible o terrenal, puesto que limpia la matriz para la infertilidad, cura maleficios y brujerías. Para el mal de ojo lo sopletean por todo el cuerpo de los niños y para enfermedades del mundo invisible inferior, para el alejamiento de los malos espíritus. El aguardiente repele los espíritus malignos función apotropaica-, los médicos tradicionales lo usan como protección para que no le entren los males del otro por su boca, además, permite expresar con más soltura y libertad lo que piensan, al vencer gracias a este el temor en quien lo consume. De otro lado, se data su uso como coadyuvante en las protestas de los esclavizados contra los esclavistas.

\section{Uso medicinal del pipilongo}

Una de las plantas que procesaron fue el pipilongo que pertenece a la familia de las piperáceas. Su nombre científico es Piper tuberculatun, su fruto tiene forma de baya (arracimado) y se utiliza en la región para condimentar las comidas. Se le adiciona al café para las personas que sufren de gastritis; las hojas frotadas en la piel sirven como repelente y también se utiliza para preparar la chicha de pipilongo.

\section{La chicha de pipilongo}

Es probable que los primeros africanos que llegaron a laborar en las haciendas del norte del Cauca tuvieran vastos conocimientos de 
procesos bioquímicos que pusieron al servicio de la economía colonial. La chicha de pipilongo es un invento de los antepasados afros en el cual utilizaban plantas que conseguían en los patios de las casas o en la finca tradicional. El pipilongo abunda en la región; el jengibre se consigue también en las fincas; el caraño que le adicionan a la mazamorra y la panela que es de uso ancestral por la misma actividad que desarrollaron en las haciendas de la región, como lo es el caso de la hacienda La Bolsa. Para fabricarla se machaca el pipilongo, caraño y jengibre, se dejan en agua durante dos días. Al tercero se le adiciona panela, se deja otro día más y se cuela. La utilizan para curar los riñones, sacar el frio del cuerpo, dolores menstruales y ciertos casos de infertilidad.

\section{Figura 4.}

Fotografía del pipilongo. Inflorescencia de pipilongo

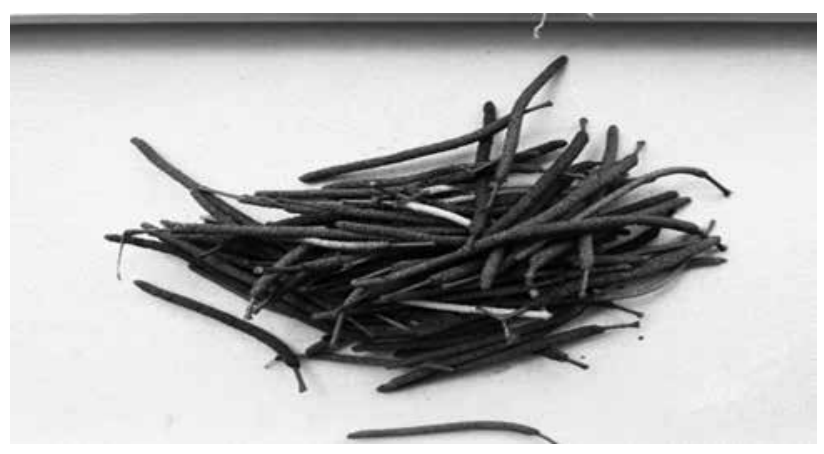

Fuente: elaboración propia 2019

Es una planta que pertenece a la familia de las piperáceas, su nombre científico es Piper tuberculatun. Es una planta que abunda en los solares de las casas y en las fincas tradicionales, en las cercas o linderos que dividen los predios. Con el pipilongo se prepara ancestralmente la chicha, pero a nivel de cocina se utiliza generalmente la inflorescencia.

\section{Sobre el pipilongo, escribió Germán Patiño} (Patiño, 2012)

En los días santos que pasaron me conecté de nuevo con la alimentación y la cocina. Pues es bien sabido que la religión tiene una influencia profunda en nuestros hábitos alimentarios y en la formación de las culturas culinarias. Tanta que el mundo se divide en dos: los que comemos carne de cerdo y los que no la consumen. Por un lado, cristianos, y por otro musulmanes, judíos y otras comunidades de creyentes. En esta preferencia o negación, nada tiene que ver la calidad de la carne del cerdo, o las creencias populares sobre su consumo. En realidad, no cocinamos cosas, sino las ideas que tenemos sobre las cosas. En un sentido extraño nos alimentamos de ideas y así cocinamos. Por ejemplo, hay ingredientes que consideramos 'prestigiosos', porque han sido habituales en las mesas de gente rica y exitosa. El caviar, por ejemplo, o la langosta. Son como la ropa de marca, valen más y están al alcance de pocos. Los demás mortales inferimos de ello que resultan exquisitos, o de calidad superior. Poco importa que no lo sean, o que en el pasado fueran alimentos habituales de gente muy pobre y poco exitosa, Como pasó efectivamente con el caviar y la langosta, alimentos originales de cerdos en el Mar Caspio, o de pescadores marginales en regiones isleñas. Lo mismo que el salmón, comida de osos o de rústicos campesinos nórdicos. Pensé en lo anterior cuando tuve la oportunidad de probar uno de los platos más tradicionales y auténticos de la cocina vallecaucana: el arroz atollado de pato con pipilongo, elaborado según la receta de doña Milvia Lucumí Carabalí. Aquí lo que llama la atención es el pipilongo, una especia maravillosa que debiera ser mucho más apreciada y valorada. Debe saberse que se trata de una variedad de pimienta, oriunda del Asia como todas las pimientas-, a la que los romanos llamaron 'piper longum', para diferenciarla de la 'piper negrum', que es la pimienta negra que comúnmente consumimos. Pero la piper longum era y es, más escasa y de sabor más sutil que la otra. De alli que también fuera mucho más costosa. En la época del Imperio Romano el gramo de piper longum podía costar hasta 30 veces más que el de pipernegrum, por lo que s[0] lo era habitual en la mesa y cocina de los nobles y de los aristócratas romanos más pudientes. Era la pimienta de los príncipes y emperadores, y el pueblo se limitaba a contemplarla de lejos. Esa misma circunstancia llevó a que su uso decayera después del descubrimiento de América, cuando este continente suministró a la cocina mundial una gran diversidad de ajíes, algunos de picantes suaves y menos iracundos, que resultaron apropiados para 
reemplazar a la antigua y costosísima piper longum. Así desapareció de la mesa romana. Pero por circunstancias desconocidas, tal vez a bordo del galeón de Manila o en las alforjas de algún monje viajero, la planta llegó a la costa pacífica colombiana y prosperó en el tremedal chocoano. Se volvió condimento de la alimentación de pueblos afrodescendientes e incluso se la utiliza con fines curativos. En el Chocó se produce un licor de pipilongo que se consume como panacea. De allí llegó a la región vallecaucana, al antiguo cantón de Caloto, y hoy los campesinos afros de Quinamayó, Timba, Robles, Suárez y otros pueblos, saborizan su cocina con esta especia de reyes, sin que nadie sepa de su vieja historia y su noble consumo.

\section{Elaboración de la chicha de pipilongo}

La chicha la elaboran con los siguientes ingredientes: 300 gramos de jengibre, 1.000 gramos de inflorescencia de pipilongo, 200 gramos de panela, 300 gramos de caraño y 3 litros de agua. Se prepara así: se machaca o macera el jengibre, el pipilongo y el caraño, se deposita en los 3 litros de agua en una olla tapada. Al siguiente día se le adiciona panela y al tercer día se cuela en un lienzo y se empaca en las botellas de vidrio con la precaución de no apretar las tapas por que se sigue fermentando y así no explota la botella.

\section{El achiote}

Se conoce también como achote, pertenece a la familia de las bixáceas y su nombre científico es Bixa orellana. Sus semillas se han utilizado ancestralmente para el tratamiento de los problemas respiratorios, también para colorear los alimentos y darles un sabor especial. Las abuelas lo preparaban en aceite, lo machacaban, le adicionaban aceite y lo sometían a cocción durante dos minutos con el objeto de conservarlo para utilizarlo en las comidas.

\section{Gandul o frijolito de año}

Es una leguminosa que trajeron las tatarabuelas africanas a las haciendas de la región. Se hacían unas trenzas en el cabello llamadas trisnejas a las que posteriormente apodaron tropas porque en ellas dibujaban las rutas de las tropas de los guerreros africanos; los españoles nunca conocieron las complejidades de esta técnica. En las tropas transportaban semillas hacia los llamados territorios libres. Una de esas semillas era la de gandul o frijolito de año como se conoce en esta región. Le llamaban el arbolito de la libertad y era, según investigadores, la comida predilecta de los esclavizados (S. de Friedemann \& Arocha, 1986).

\section{Jengibre}

Planta de la familia zingiberáceas cuyo nombre botánico es Zingiber officinali, tradicionalmente se ha utilizado en esta región como un estimulante gastrointestinal, expectorante y el principal uso es diurético. Se utiliza su raíz. Cabe destacar que en esta región las plantas empleadas en la cocina tienen dos funciones principales: servir como condimento para saborisarlas y una función terapéutica, ya sea a nivel preventivo o de control de ciertas enfermedades.

\section{El caigamos juntos, síntesis de creatividad}

Figura 5.

Fotografía del caigamos juntos

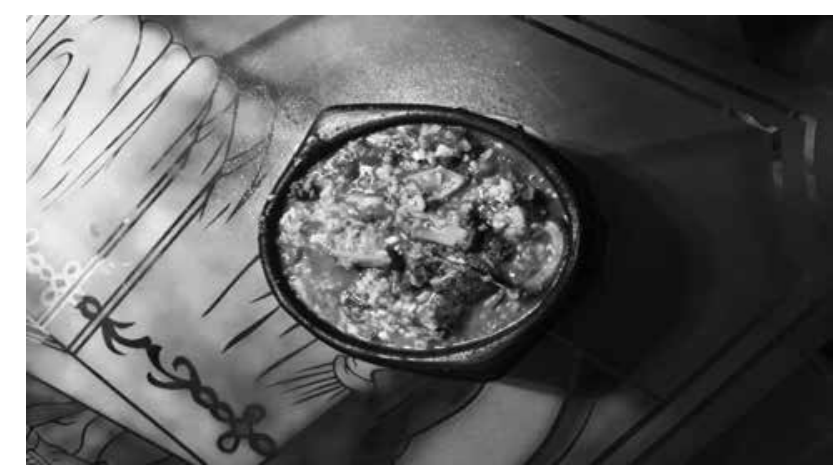

Fuente: elaboración propia (2019) 
Es un plato propio de esta región cultural preparado con los restos del mercado de la semana cuando todo se está acabando. La creatividad de estas mujeres las llevó a inventar este plato, al cual le echan un poco de cada cosa. Por ejemplo, un poco de arroz, gandul o frijolito de año, frijol, arverja, plátano, rascadera o mafafa, arracacha; condimentos como azafrán de vena, achiote, pipilongo, jengibre, cimarrón, cilantro y callo o tripa y hueso carnudo. Vale resaltar que los ingredientes proteínicos animales tales como el callo o tripa son de muy bajo costo, lo que justifica su utilización en tiempo de escases económica. Para prepararlo se necesita lo siguiente:

\section{Tabla 5.}

Ingredientes del caigamos juntos

\begin{tabular}{|c|c|}
\hline \multicolumn{2}{|c|}{$\begin{array}{l}\text { INGREDIENTES DEL CAIGAMOS JUNTOS } \\
\text { Preparación para } 5 \text { personas }\end{array}$} \\
\hline 1.5 litros de agua & Aceite \\
\hline Sal al gusto & 125 gramos de arroz \\
\hline $\begin{array}{l}1 \text { libra de cayo o tripa de } \\
\text { res }\end{array}$ & $\begin{array}{l}25 \text { gramos de frijolito de } \\
\text { año }\end{array}$ \\
\hline 1 plátano & 25 gramos de alverja \\
\hline $\begin{array}{l}2 \text { inflorescencias de } \\
\text { pipilongo }\end{array}$ & 50 gramos de arracacha \\
\hline 5 gramos de jengibre & 10 gramos de achiote \\
\hline \multicolumn{2}{|c|}{ Inv. Carlos Alberto Velasco Díaz, PhD } \\
\hline
\end{tabular}

Fuente: el autor

Un día antes de la preparación se dejan en agua los frijoles a utilizar (gandul, alverja y frijol). Se pone la olla al fuego con el callo o tripa con sal (en tiempos modernos se utiliza olla a presión). Cuando ya ablande se adiciona una cucharada de cimarrón finamente picado, la inflorescencia o fruto de pipilongo, el jengibre machacado para darle el toque especial y el orégano. Luego se añaden los frijoles remojados desde el día anterior. Pasados unos 15 minutos y que los componentes estén blandos se adiciona el arroz y cuando este esté blando se agrega el plátano verde picado a uña, media taza de cebolla larga finamente picada con cilantro y media taza de chicha de pipilongo. Cuando el arroz esté blando y el caigamos juntos haya tomado consistencia de atollado se retira la olla del fuego y se sirve con hogao.

\section{Preparación del hogao afrocaucano}

En un cuarto de taza de aceite se echa achiote diluido y sal, allí se ponen a cocinar media taza de cebolla larga o junca y cinco dientes de ajo finamente picados. Se añade media taza de pimentones finamente picados y media taza de tomates picados. Se deja cocinar muy bien a fuego lento y se esparce sobre los platos del caigamos juntos al momento de servir.

\section{A manera de conclusión}

En el marco de indagaciones hechas para el proyecto de etnoeducación e interculturalidad se pudieron analizar muchas de las sabidurías que mueven las comunidades étnicas alrededor de la cocina, vista como producto de la cultura y no simplemente como un recetario. La cocina da cuenta de los procesos socioculturales de las comunidades afrocolombianas. En este proceso se identificó una terminología que se deja en tensión, se cuestionó el uso del término gastronomía porque la comida no se disfruta en la región gástrica, se aprecia con los sentidos, boca, vista y olfato. Además, alrededor del fogón se tejen historias, se compone música y se hace trasmisión corporal de los saberes ancestrales.

Las mujeres africanas, en su inteligencia y creatividad durante la ruta trasatlántica, utilizaron muchas estrategias para la reconstrucción de la vida en el nuevo continente. Llevaban arroz y granos 
escondidos en sus cabellos, es decir que para las mujeres del pacífico potamoral no era nueva la práctica de camuflar semillas y oro en sus trisnejas o trenzas para pagar las manumisiones de sus maridos e hijos. Desde esta perspectiva se puede plantear que en la diáspora no solo llegaron seres, sino también saberes.

\section{Referentes de poder 0 códigos vivos}

Diego Luis Gómez Viáfara, de 45 años (q.e.p.d.), residía en el municipio de Villa Rica Cauca.

Bárbara Morales, de 76 años (q.e.p.d.), residía en el municipio de Villa Rica Cauca.

Nemesia Mina, de 80 años residente en el municipio de Santander de Quilichao, norte del Cauca.

Petronila Viáfara de 75 años (q.e.p.d.), de la comunidad de Quinamayó, sur del Valle del Cauca.

Ana Tulia Olaya "Manato", de 90 años (q.e.p.d.) de la comunidad de Villa Rica norte del Cauca.

\section{Referencias}

Banguero Velasco, R. (s.f.). Territorialidad en los reales de minas del norte del Cauca. Doctorado en Humanidades. Universidad del Valle, Cali.

Carney, J., \& Acevedo Marín, R. (2003). Plantas de la Diáspora Africana en la botánica americana de la fase Colonial. Memoria y sociedad, 8-23.

Guerrero Arias, P. (2018). La chakana del corazonar, desde las espiritualidades y las sabidurías insurgentes de la Abya Yala. Cuenca Ecuador: Editorial Universitaria Abya Yala.
Otro elemento de gran factura en este análisis tiene que ver con que estas culturas construyeron cosmocimientos -no conocimientos-, en medio de la negación hecha de su humanidad y derechos mínimos. Muy probablemente este proceso se dio porque hubo en sus habitantes unos procesos de resiliencia que les han permitido superar todas las situaciones lastre que desde tiempos coloniales padecen.

Max-Neef, M. (20 de Julio de 2019). https://es.scribd. com/document/220732728/El-Acto-Creativo-Manfred-Max-Neef. Obtenido de https:// docplayer.es/22137522-El-acto-creativo-pormanfred-max-neef-1.html

Max-Neef, M., Elizalde, A., \& Hopenhayn, M. (1986). Desarrollo a escala humana, una opción para el futuro. Chile: Cepaur.

Patiño Ossa, G. (2007). Fogón de negros, cocina y cultura en una región latinoamericana. Bogotá: Printed in Colombia.

Ramos D, L. G. (2014). Medicina tradicional de las comunidades afrocolombianas del norte del Cauca. Puerto Tejada: Litografía Kreart.

Rosario, J., \& Carrion, J. (1951). El negro. Puerto Rico: Universidad de Puerto Rico.

S. de Friedemann, N., \& Arocha, J. (1986). De sol a sol: génesis, transformación y presencia de los negros en Colombia. Bogotá: Planeta Colombiana Editorial S.A.

Velasco Díaz, C. A. (2011). Comunidad, cultura y etnoeducación afrocolombiana. Cali: Facultad de Humanidades . 